\title{
Nuclear receptors from the ctenophore Mnemiopsis leidyi lack a zinc-finger DNA-binding domain: lineage-specific loss or ancestral condition in the emergence of the nuclear receptor superfamily?
}

Adam M Reitzel', Kevin Pang ${ }^{2}$, Joseph F Ryan ${ }^{3}$, James C Mullikin ${ }^{3}$, Mark Q Martindale ${ }^{2}$, Andreas D Baxevanis ${ }^{3}$, Ann M Tarrant ${ }^{1 *}$

\begin{abstract}
Background: Nuclear receptors (NRs) are an ancient superfamily of metazoan transcription factors that play critical roles in regulation of reproduction, development, and energetic homeostasis. Although the evolutionary relationships among NRs are well-described in two prominent clades of animals (deuterostomes and protostomes), comparatively little information has been reported on the diversity of NRs in early diverging metazoans. Here, we identified NRs from the phylum Ctenophora and used a phylogenomic approach to explore the emergence of the NR superfamily in the animal kingdom. In addition, to gain insight into conserved or novel functions, we examined NR expression during ctenophore development.

Results: We report the first described NRs from the phylum Ctenophora: two from Mnemiopsis leidyi and one from Pleurobrachia pileus. All ctenophore NRs contained a ligand-binding domain and grouped with NRs from the subfamily NR2A (HNF4). Surprisingly, all the ctenophore NRs lacked the highly conserved DNA-binding domain (DBD). NRs from Mnemiopsis were expressed in different regions of developing ctenophores. One was broadly expressed in the endoderm during gastrulation. The second was initially expressed in the ectoderm during gastrulation, in regions corresponding to the future tentacles; subsequent expression was restricted to the apical organ. Phylogenetic analyses of NRs from ctenophores, sponges, cnidarians, and a placozoan support the hypothesis that expansion of the superfamily occurred in a step-wise fashion, with initial radiations in NR family 2, followed by representatives of NR families 3, 6, and 1/4 originating prior to the appearance of the bilaterian ancestor.

Conclusions: Our study provides the first description of NRs from ctenophores, including the full complement from Mnemiopsis. Ctenophores have the least diverse NR complement of any animal phylum with representatives that cluster with only one subfamily (NR2A). Ctenophores and sponges have a similarly restricted NR complement supporting the hypothesis that the original NR was HNF4-like and that these lineages are the first two branches from the animal tree. The absence of a zinc-finger DNA-binding domain in the two ctenophore species suggests two hypotheses: this domain may have been secondarily lost within the ctenophore lineage or, if ctenophores are the first branch off the animal tree, the original NR may have lacked the canonical DBD. Phylogenomic analyses and categorization of NRs from all four early diverging animal phyla compared with the complement from bilaterians suggest the rate of NR diversification prior to the cnidarian-bilaterian split was relatively modest, with independent radiations of several NR subfamilies within the cnidarian lineage.
\end{abstract}

\footnotetext{
* Correspondence: atarrant@whoi.edu

'Biology Department, Woods Hole Oceanographic Institution, Woods Hole, MA, USA

Full list of author information is available at the end of the article
} 


\section{Background}

Nuclear receptors (NRs) are a class of transcription factors that regulate diverse developmental and physiological processes in animals. The characteristic domains of NRs are the DNA-binding domain (DBD), which includes a Cys-Cys zinc coordinating region, and the ligand-binding domain (LBD), a carboxy-terminal domain that binds ligands and coactivators for modulation of NR function, facilitates receptor dimerization, and contains an activation function. Current evidence has shown that NRs are restricted to animals because they are present in all metazoan phyla that have been surveyed but not in plants or fungi [1-6]. NR-like transcription factors have been identified in some fungi based on a region that has low conservation with the LBD of animal NRs; however, whether these are evolutionary related to NRs in animals is not known [7].

Genes in the NR superfamily are classified into six major families (NR 1 through NR 6; Nuclear Receptor Nomenclature Committee [8]). All six families (and many subfamilies) are represented in protostomes and deuterostomes, supporting the conclusion that NRs had diversified prior to the bilaterian ancestor [1]. Thus, characterizing NRs in species representative of the early diverging animal phyla would provide critical information that would allow for a better understanding of the evolutionary history and emergence of this superfamily. NRs have been reported from three of the four classes of cnidarians (Anthozoa [3,5], Cubozoa [9], and Hydrozoa [2]), including the full complement from the sea anemone Nematostella vectensis [5], two sponges $[4,6,10]$, and one placozoan $[11,12]$. Phylogenetic analyses of these NRs have shown that most of these genes belong to NR family 2 . The exceptions are: (1) one NR family 3 member from the placozoan Trichoplax adhaerens, (2) one ortholog to NR family 6 from the cnidarian Nematostella, and (3) three genes from Nematostella that form an outgroup to NR families 1 and 4. Taken together, these data suggest that the evolution of NRs may be complex and, more importantly, that early diverging metazoans are the appropriate groups to study in order to understand how and when the NR superfamily has evolved and diversified.

Among the early branching animal lineages, NRs have not been identified or described in any species from Phylum Ctenophora. The apparent phylogenetic position of ctenophores in relation to other animal phyla has varied among studies, with recent analyses placing them at the base of the animal tree $[13,14]$, as a sister clade with other non-bilaterians to the Bilateria [15], in one clade with cnidarians [16], or as an outgroup to a clade of the Placozoa, Cnidaria, and Bilateria $[17,18]$ (see [19] for a summary). Determining the correct phylogenetic position of ctenophores and other metazoan phyla is critical for accurately reconstructing the evolutionary history of individual gene families. Reciprocally, accumulated analyses comparing the diversity of multiple gene families among early diverging phyla may help investigators to select among the particular phylogenetic hypotheses. Characterizing the NR complement from a ctenophore species is a constructive step towards understanding the evolution of the superfamily and, at the same time, it provides some insight into the relationship of ctenophores to other metazoans. Similarly, comparing the expression of NRs in ctenophores with other species would provide data needed to assess potential conservation of the spatial and temporal expression of these transcription factors.

Here, we describe NRs from two ctenophores, the lobate Mnemiopsis leidyi and the tentaculate Pleurobrachia pileus. These two species are from different orders and, thus, represent distant lineages within the Ctenophora [20]. Additionally, for Mnemiopsis, we describe the intron-exon structure and the spatio-temporal developmental expression of these NRs using in situ hybridization. We then applied phylogenetic methods to characterize NR diversity from 12 early diverging metazoans (two sponges, two ctenophores, one placozoan, and seven cnidarians) and developed a hypothesis for the early diversification of the NR superfamily.

\section{Methods}

\section{Identification and annotation of Mnemiopsis leidyi} nuclear receptors

We identified candidate NRs through BLAST queries of the assembled genome and gene models for Mnemiopsis leidyi (physical coverage of genome approximately $50 \times$, see [21]). For these searches we used a diverse set of fulllength NRs from the sponge Amphimedon queenslandica, the anemone Nematostella vectensis, Homo sapiens, and Drosophila melanogaster. Through these similarity searches we identified two matches that were reciprocal best BLAST hits with other animal NRs, showing strong similarity to the LBD. No BLAST queries returned any ctenophore gene model or region of the genome with similarity to the DBD. TBLASTN searches of the Mnemiopsis genome and BLASTP searches of the proteome using only the DBD from sponge and anemone HNF4 resulted in weak matches that were on separate contigs from the two predicted genes from Mnemiopsis with high similarity to the LBD. The top ctenophore protein match using the DBD from sponge HNF4 (E-value $=0.027$ ) was BLASTed to human refseq and exhibited greatest, although low, similarity to members of the LIM family. HMM searches of the ctenophore proteome failed to identify any additional matches. 
For the two candidate NRs, we performed 5' - and 3'-RACE with gene specific primers, using cDNA prepared from RNA pooled from diverse developmental stages (see Additional file 1 for all primers). RACE products were cloned into pGEMT (Promega, Madison, WI, USA) and sequenced. Overlapping fragments were assembled in silico to produce the complete transcript. To confirm the two gene products, we amplified and sequenced the entire open reading frame for each NR. Each primer pair yielded a single product that matched the conceptually assembled fragment. We characterized gene structure for each Mnemiopsis NR by aligning the full length transcripts to the assembled genome. We also identified a NR in a second species of ctenophore, Pleurobrachia pileus, through BLAST queries of ESTs in GenBank. We assembled a full-length transcript for a single Pleurobrachia NR by assembling overlapping ESTs (GenBank accession numbers FP998505, FP993827, FP993707, and FP997412).

\section{Developmental expression of Mnemiopsis nuclear receptors}

Whole-mount in situ hybridizations were performed as previously described [22]. A RACE product for MlNR1 (approximately $1,000 \mathrm{bp}$ ) and the full open reading frame for $M l N R 2$ (1,137 bp) were used as templates to transcribe digoxigenin-labeled antisense RNA probes (Megascript Kit, Ambion, Austin, TX, USA). Developmental stages from gastrulation through the early cydippid were probed for spatial expression.

Phylogenetic analyses of the nuclear receptor superfamily Through a combination of literature searches and BLAST queries, we assembled a dataset of $54 \mathrm{NRs}$ from early diverging animals in the phyla Ctenophora, Porifera, Placozoa, and Cnidaria (Accession numbers given in Additional file 2). We identified three additional NRs from the coral Acropora millepora [data from 23] which were combined with previously described NRs from this species [3]. NRs from two cnidarians (Metridium senile $(\mathrm{n}=2)$, Hydra magnipapillata $(\mathrm{n}=6))$ were identified through BLAST queries against GenBank. Sequences from Homo sapiens, Drosophila melanogaster, and Caenorhabditis elegans from Bertrand et al. [1] were used as representative bilaterian sequences.

Full-length sequences for all taxa were aligned with Muscle 3.6 [24] and edited manually in the case of clear errors. For some taxa, only partial sequences were available, containing only the DBD in many cases. Due to the absence of a well-conserved DBD in the three ctenophore sequences, these proteins were manually corrected in an effort to optimize the alignment.

Maximum likelihood analyses were conducted with RAxML (version 7.0.4, [25]) and Bayesian analyses with
MrBayes v.3.1.2 [26] using a JTT $+\mathrm{G}+\mathrm{F}$ matrix (model determined by RAxML model picker) and a trimmed alignment containing the DBD and most of the LBD, beginning in helix 3. Support for particular nodes for maximum likelihood analyses was assessed with 1,000 bootstraps. For the Bayesian analysis, two independent analyses were performed with five chains run for five million generations and sampled every 500 generations. The first 1.25 million generations were discarded as burn-in. Log likelihood values were plotted and found to be asymptotic well before the burn-in fraction. Trees were visualized and illustrated with FigTree v1.1.2 (http://tree.bio.ed.ac.uk/software/figtree/).

\section{Results}

Nuclear receptors from the ctenophores Mnemiopsis leidyi and Pleurobrachia pileus

BLAST queries of the Mnemiopsis genome and gene models resulted in two hits with significant similarity to the LBD of NRs from diverse metazoans. Assembled RACE products contained the complete open reading frame of each NR, as well as 5'- and 3'-UTR sequence (protein sequences are given in Additional file 3). MlNR1 is encoded by a transcript 1,408 bp long with an open reading frame of 268 amino acids. MlNR1 is composed of eight exons spanning almost $10 \mathrm{~kb}$ of genomic sequence, with the coding sequence on seven exons (Figure 1A). We identified a polyA tail 508 bp downstream from the stop codon and $156 \mathrm{bp}$ of 5 '-UTR. MlNR2 is encoded by a transcript 1,650 bp long with an open reading frame of 379 amino acids. MlNR2 is a single-exon gene with no intervening introns. The 3'-RACE sequence of MlNR2 contained a polyA tail $357 \mathrm{bp}$ downstream of the stop codon. The 5'-RACE sequence contained 123 bp of UTR. By comparison, the assembled NR from Pleurobrachia ESTs had an open reading frame of 484 amino acids. We did not identify a polyA tail in the assembled transcript.

Neither of the two NRs from Mnemiopsis nor the single NR from Pleurobrachia contained a conserved zinc-finger DNA-binding domain (DBD) typical for NRs across the superfamily. The optimized alignment of the aminoterminal of the ctenophore NRs with the DBD from HNF4 from other animals suggests that this region in the ctenophore proteins contains very little conservation and is interrupted by insertions (Figure 1B). It was not possible to identify the conserved cysteines characteristic of zinc-finger motifs within the ctenophore sequences. The amino-terminal region of the ctenophore NRs failed to consistently match any protein or domain when BLASTed at NCBI. Despite the absence of a conserved DBD, each of the ctenophore NRs contains an LBD that aligned reasonably well with LBDs from other NRs (Figure 1C). Two of the ctenophore NRs (MlNR2 and 


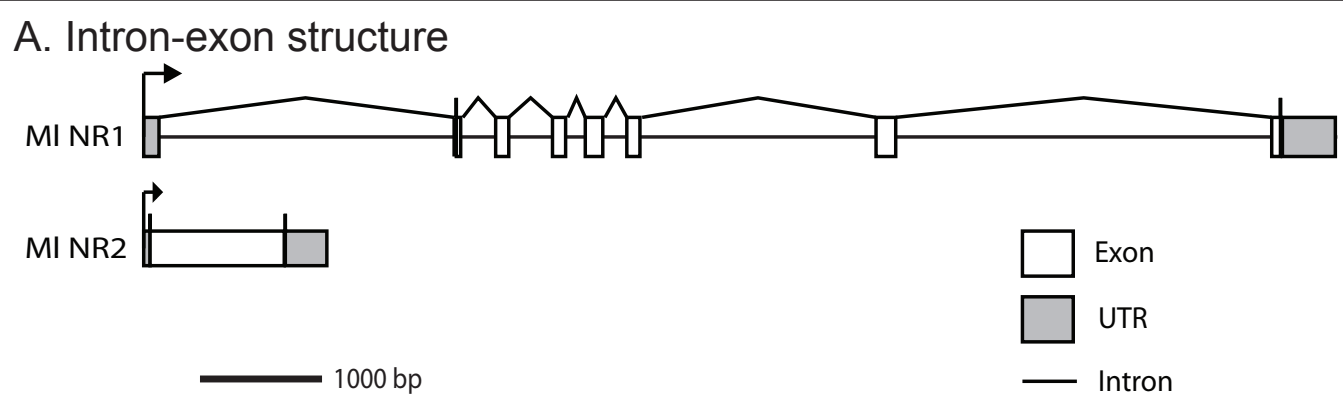

\section{B. DNA-binding domain}

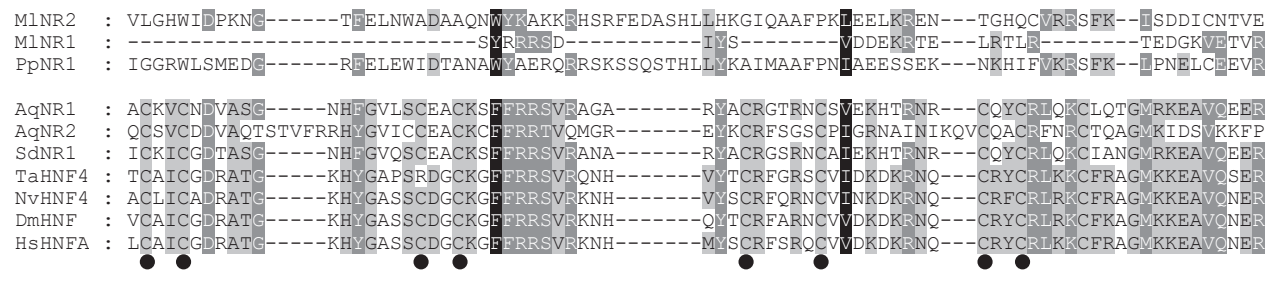

\section{Ligand-binding domain}

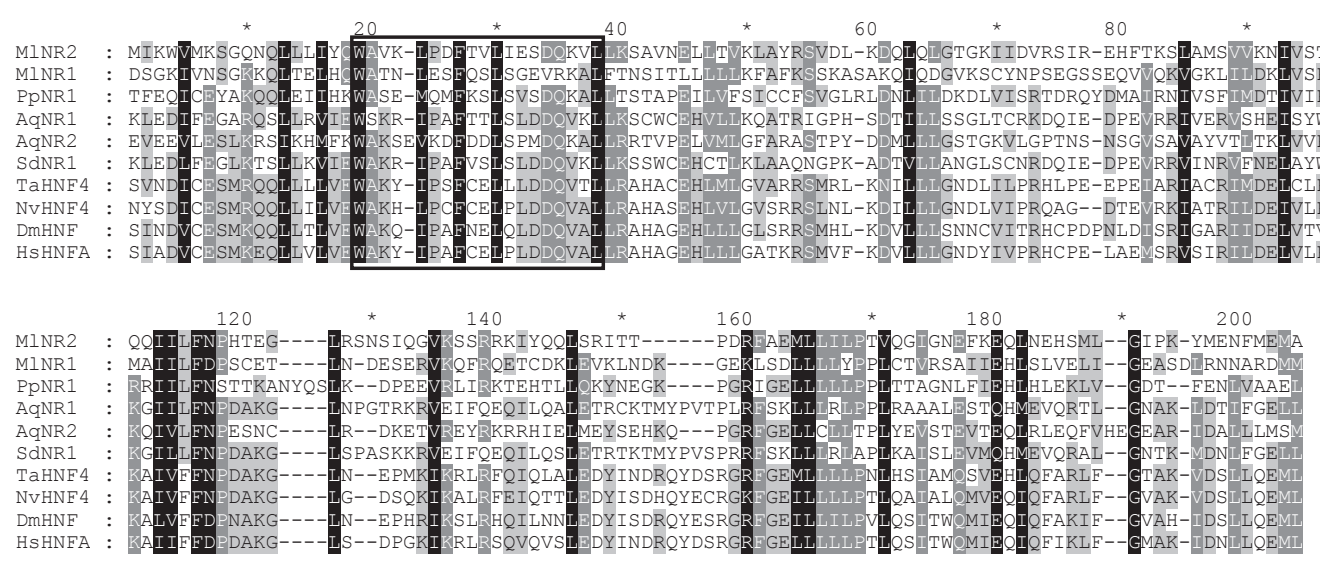

Figure 1 Nuclear receptors from the ctenophores Mnemiopsis leidyi and Pleurobrachia pileus. (A) Intron-exon structure of the two nuclear receptors from Mnemiopsis. MINR2 is a single exon gene. MINR1 has a more complex intron-exon structure with eight exons, seven of which code for the inferred open-reading frame. (B) Alignment of the amino-terminal region of the ctenophore NRs with the DNA-binding domains of NRs from two sponges (Amphimedon queenslandica (Aq) and Suberites domuncula (Sd)), and HNF4 from Trichoplax adhaerens (Ta), Nematostella vectensis (Nv), Drosophila melanogaster (Dm), and Homo sapiens $(\mathrm{Hs})$. The ctenophore proteins align poorly, including an absence of the conserved cysteines (indicated by black circles), and an optimized alignment contains insertions and deletions relative to the DBD of other animals. (C) Alignment of the ligand-binding domain from the same taxa as in (B). The ctenophore LBD is well-conserved, particularly the nuclear receptor signature motif spanning helix 3 and 4 (boxed).

PpNR1) have the NR signature motif (boxed region in Figure 1C), whereas $M l N R 1$ has retained only a portion of this sequence. The relative conservation of the LBD in the ctenophore NRs was similar to that of NRs previously reported from two sponges $[4,6,10]$ when compared with bilaterian HNF4 (approximately $30 \%$ identity, approximately $50 \%$ similarity).

\section{Developmental expression of Mnemiopsis nuclear receptors}

Using in situ hybridization, we documented the developmental expression of $M l N R 1$ and MlNR2. MlNR1 was first expressed during midgastrulation in the ectoderm in regions of future tentacle development (Figure 2A, B, $F, G)$. Later in embryogenesis, expression continued in 


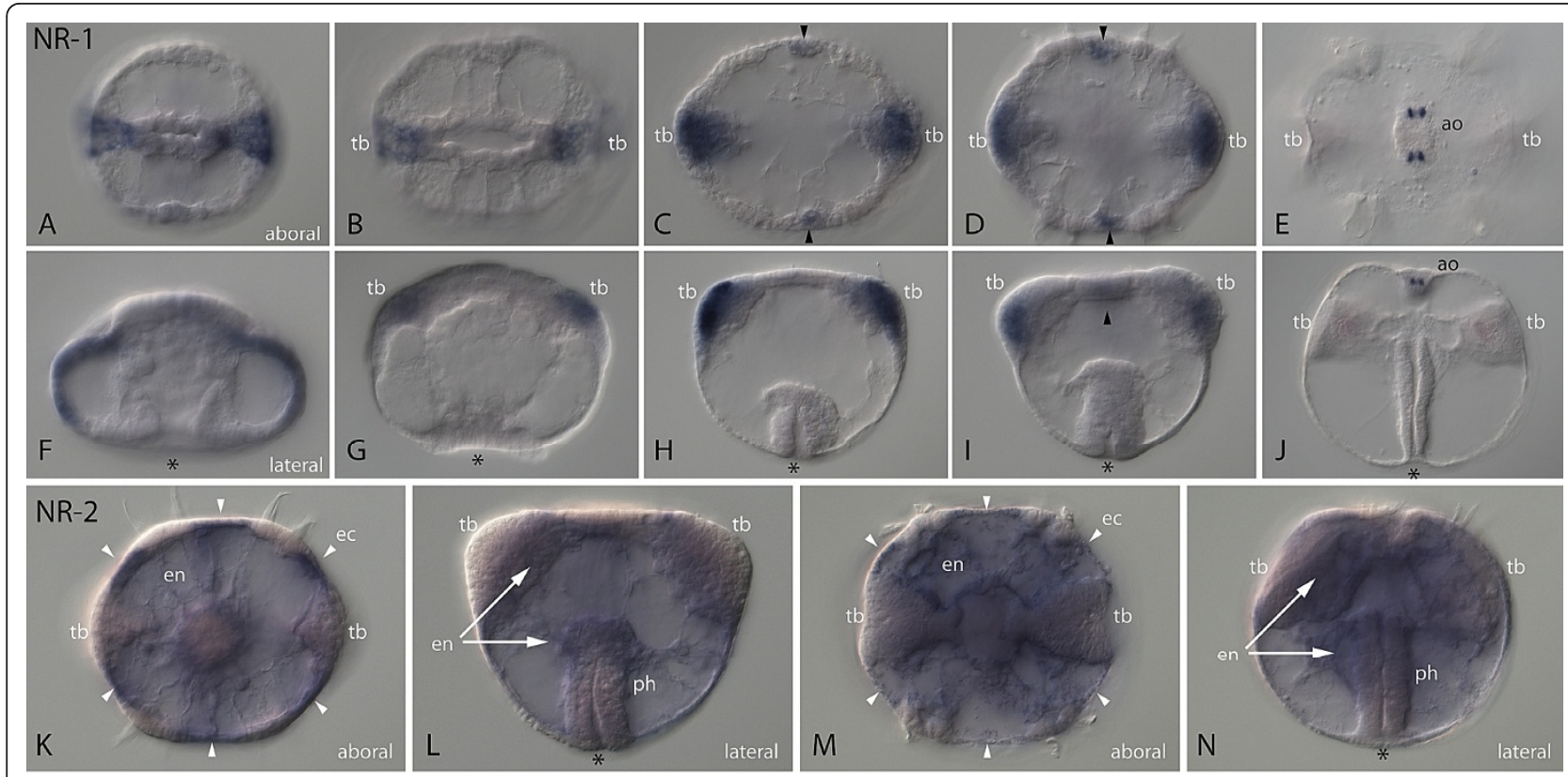

Figure 2 Expression of MINR1 (A-J) and MINR2 (K-N) in embryos and juveniles of Mnemiopsis. A-E show aboral views of MINR1, with F-J showing lateral views of the corresponding embryo. (A-B, F-G) MINR1 expression in ectoderm of the future tentacle bulb (tb) in the gastrula and late gastrula stage. (C-D, $\mathbf{H}-\mathbf{I})$ Expression continues in ectoderm of future tentacle bulb as well as in additional domain along the sagittal plane (arrowhead) in the postgastrula. (E, J) MINR1 expression is restricted to the apical organ (ao) in the cydippid stage. (K-N) MINR2 is broadly detected in the endoderm (en) in developmental stages after gastrulation and in the cydippid. It is only in the most aboral part of the pharynx (ph). There are also additional domains in the ectoderm (white arrowheads).

the same location, as well as in an additional adoral domain along the sagittal plane between the adpharyngeal comb rows (Figure 2C, D). In the cydippid stage, expression of MlNR1 was restricted to four discreet domains of the floor of the apical organ flanking the sagittal plane (Figure 2E, J). These were not associated with the balancing cilia that support the mineral containing lithocytes. MlNR2 was expressed broadly in the endoderm and parts of the ectoderm after gastrulation and in the cydippid (Figure $2 \mathrm{~K}-\mathrm{N}$ ). Expression of $M I N R 2$ was not observed earlier in gastrulation.

\section{Phylogenetic position of ctenophore NRs and distribution of NRs from early diverging metazoans}

Maximum likelihood analyses of the NRs from the selected taxa spanning the animal kingdom reproduced monophyletic relationships for all six recognized NR families (Figure 3). The bootstrap support for these families varied from $97 \%$ for NR family 1 to $52 \%$ for NR family 6 . Previous studies have shown similarly low support for NR family 6 and for the node including this family and its sister family, NR family $5[5,27,28]$. Bayesian analyses resulted in a largely identical topology as the maximum likelihood analysis, with high posterior probabilities (0.93 to 1) for each NR family (Additional file 4).

The two NRs from Mnemiopsis and the one from Pleurobrachia cluster with the HNF4 subfamily (NR2A) and share greatest sequence similarity with HNF4s from other animals including Trichoplax, diverse cnidarians, and bilaterians. This cluster also contains a NR recently identified in the sponge Amphimedon [10]. The sponges Amphimedon and Suberites also contain a second NR-type identified by Larroux et al. [4] and Wiens et al. [6], respectively, that forms a sponge-specific NR cluster that groups between the HNF4 subfamily and all other nuclear receptors. These results suggest that ctenophores and sponges have representatives that most closely group with only one NR subfamily (NR2A) but that sponges contain an additional type of NR not present in any other species sampled to-date. Thus, ctenophores contain the most restricted NR complement of any species yet described.

For the placozoan Trichoplax adhaerens, we identified four NRs, consistent with results published earlier (Supplemental Figure S9.13 in [11]). Trichoplax NR1 grouped in NR family 2, with weak support in subfamily NR2F. Two additional NRs were strongly supported as orthologs to HNF4 (NR2A) and RXR (NR2B). Trichoplax NR4 was a strongly supported member of NR family 3 . This result supports a previous report showing a NR 3 member from Trichoplax [12]. This earlier study concluded that this NR was likely an ERR ortholog. In our analysis, we found that this Trichoplax NR is a member of NR family 3 , but with no particular relationship to any of the NR 3 subfamilies. 


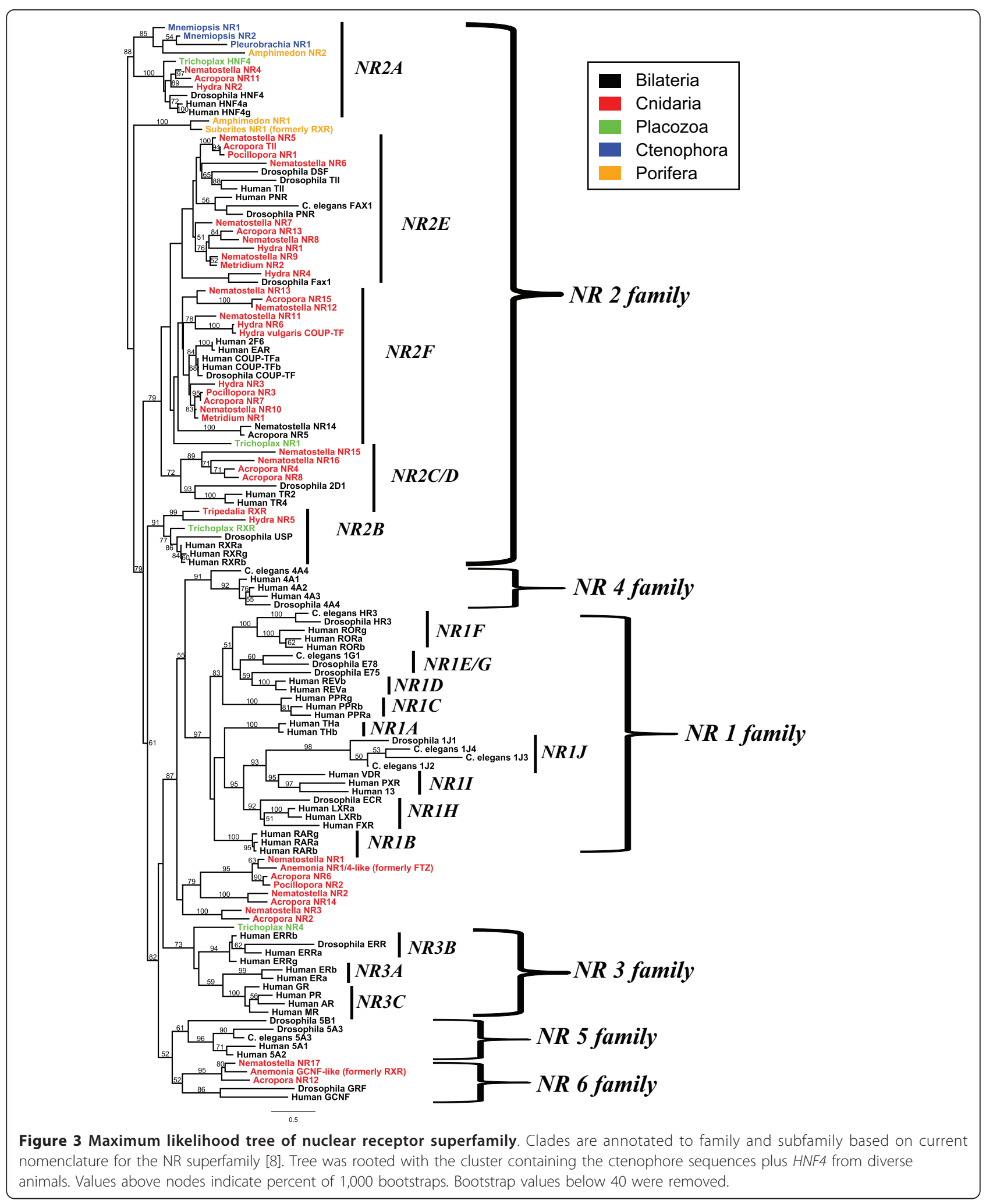


For the cnidarians, we analyzed NRs from five anthozoans, one cubozoan, and one hydrozoan. From the anthozoans, a previous study reported that the NR complement of Nematostella vectensis contained 17 NRs [3]. While most of the Nematostella NRs share wellsupported orthologs with Acropora, orthologs of several of the Nematostella NRs have not yet been identified in Acropora or other corals. Nematostella sequences without clear Acropora counterparts are all sequences in subfamilies with coral orthologs, likely representing either paralogs potentially resulting from lineage-specific gene duplication or genes not yet sequenced from the coral. Three previously published NRs from the coral Pocillopora damicornis [29] grouped with tailless (NR2E), COUP-TF (NR2F), and the cnidarian specific NR1/4 clade. We identified two NRs from an EST collection for the anemone Metridium. These NRs grouped within family NR2E and 2F; one was orthologous to bilaterian COUP-TFs, and the other clustered with a cnidarian-specific NR radiation within family NR2E. We also included two previously published sequences from Anemonia [28] that grouped well with the anthozoan radiation of NR family $1 / 4$ and a GCNF, despite their previous annotation as an FTZ and $R X R$, respectively. Together, the combined NR data show that anthozoans contain representatives of four of the five NR 2 subfamilies, with no ortholog of $R X R$ (NR2B) in any species. Additionally, we identified three species (Nematostella, Acropora, and Anemonia) having an NR that groups most closely with GCNF (NR6), consistent with previous studies $[5,10]$. However, the support for these cnidarian genes with NR6 was modest and these genes may instead represent NR5/6 orthologs. Finally, in four anthozoan species, we observed at least one NR that groups in a cnidarian-specific cluster that was positioned as an outgroup to NR families 1 and 4, which only contain NRs from bilaterians. We originally identified three NRs from this group in Nematostella, leaving open the question as to whether these genes were unique to this anemone as a result of a species-specific duplication and divergence, or if these were more broadly represented in anthozoans. By including additional anthozoan genes, we identified well-supported coral orthologs to each Nematostella gene, bolstering the conclusion that this group is diverse within the class Anthozoa.

We identified six NRs from the recently completed genome of the hydrozoan Hydra magnipapillata [30]. One of these NRs (HmNR6) grouped strongly with one previously reported COUP-TF (NR2F) gene from the congener $H$. vulgaris [2]. These two NRs group in a separate cluster (with other cnidarian members of subfamily NR2F), but more distantly than with COUP-TFs from bilaterians and more closely related homologs from cnidarians, including an NR from $H$. magnipapillata
(HmNR3). This suggests that these two NRs from two Hydra species are retained duplicates from an earlier cnidarian radiation in this family, a result consistent with that found by Escriva et al. [31]. The other NRs from $H$. magnipapillata were all supported as members of the NR2 family. HmNR2 was supported as an HNF4 family member. HmNR1 and HmNR4 group as members of the NR2E subfamily, although the position of $H m N R 4$ varied within the NR2 family among analyses. The last NR from $H$. magnipapillata (HmNR5) grouped with strong support as an ortholog to $R X R$, grouping with $R X R$ s from bilaterians and the placozoan Trichoplax. This NR is only the second $R X R$ identified from the Cnidaria, with a previous report of a $R X R$ from the cubozoan jellyfish Tripedalia [9]. Thus, $R X R$ orthologs have only been reported from the medusozoan clade within the phylum Cnidaria. We did not identify an ortholog to NR family $1 / 4$ or 6 in $H$. magnipapillata. Finally, we found no NR from $H$. magnipapillata or any anthozoan with support as a member of the NR family 3.

\section{Discussion}

By characterizing the first NRs from the phylum Ctenophora and assembling NR sequences from diverse early diverging animals, we have gleaned new insights into the evolution of this superfamily of transcription factors. The ctenophore sequences in particular provoke new hypotheses about the origin of this gene superfamily and the evolution of the classical NR gene structure.

\section{Ctenophore NRs lacking a DBD}

The zinc-finger DBD is typically the mostly conserved feature of NRs throughout the superfamily. The two NRs from Mnemiopsis and the one from Pleurobrachia have a conserved LBD, but all of these lacked a DBD typical of NRs. The two NRs from Mnemiopsis are supported as paralogs due to a duplication event somewhere in the ctenophore lineage. One of the Mnemiopsis NRs (MlNR2) is coded by a single exon; this is evidence of a potential retroposition event [32]. The ctenophore genes are the first NRs lacking a DBD from any non-bilaterian animal. We failed to identify any matches to a DBD elsewhere in the genome. The lack of a DBD and the potential position of ctenophores as the first branch from the animal tree suggest two competing hypotheses: the DBD was lost within the ctenophore lineage from an ancestral NR with both the $\mathrm{DBD}$ and LBD, or the ancestral NR contained only the LBD and the zinc-finger DBD was added later in animal evolution.

If the absence of the DBD is due to domain loss, it is likely to be broadly represented in ctenophores because Mnemiopsis and Pleurobrachia are members of separate orders - the Lobata and Cydippida, respectively [20]. 
The NRs reported from the three other early diverging phyla (Porifera, Placozoa, and Cnidaria) all contain both a DBD and an LBD. Within the bilaterians, NR domain structure has been modified in particular lineages, including duplication of the DBD in a novel protostome NR subfamily [33], loss of the DBD from vertebrate DAX1 (NR0B1) and SHP (NR0B2) [34,35], and loss of a conserved LBD in some NRs from insects (for example, knirps [36]) and nematodes (for example, odr-7 [37]). Thus, although loss of the DBD in ctenophores would be unique among early diverging animals, similar modifications of NR domains have occurred in other animals.

A more provocative but equally supported hypothesis for the absence of a canonical DBD in ctenophore NRs is that the ancestral NR contained only an LBD and the DBD was added later in animal evolution. This hypothesis is contingent on (1) the phylogenetic placement of ctenophores as the first branch of the animal tree, which has been shown in previous phylogenomic analyses $[13,14]$ yet remains controversial $[16,18]$, and (2) NRs from other ctenophore species lacking a zinc-finger DBD, like the distantly related species reported here. NRs are unique to animals, but several recent studies have shown "NR-like" genes in fungi [38]. These proteins (for example, Oaf1 and Pip2 from the yeast Saccharomyces cerevisiae [7]) have regions of structural similarity to animal NR LBDs but not zinc-finger DBDs, function as protein dimers, and bind fatty acids as ligands which regulate their function.

\section{Potential function of ctenophore nuclear receptors}

Expression patterns of NRs from Mnemiopsis were consistent with NRs having a role in development. MlNR1 was expressed in spatially restricted portions of the ectoderm during gastrulation, corresponding to the position of the future tentacles. Subsequent expression in the cydippid stage was confined to the apical organ. However, MlNR2 was expressed broadly in the endoderm and portions of the ectoderm after gastrulation and in the cydippid stage. Previous studies with HNF4 in the sponge Amphimedon showed ubiquitous expression throughout development [10]. Spatial expression of HNF4 genes has not been reported from any cnidarian or the placozoan Trichoplax. However, HNF4 from the anemone Nematostella was expressed at high levels throughout developmental and adult stages, suggesting potential roles in both development and in normal cell physiology [5].

Nuclear receptors regulate the transcription of downstream genes, primarily through binding to specific DNA-responsive elements [39]. Due to the absence of a conserved DBD, the ctenophore NRs are unlikely to act as transcription factors by binding DNA-responsive elements typical of other NRs. The amino-terminal regions of these ctenophore NRs contain no conserved domains similar to other transcription factors; thus, we have no a priori expectation for what DNA motifs these proteins may bind (if any). Despite lacking a zinc-finger DBD, the mammalian NR DAX-1 binds DNA by recognizing hairpin structures [40] rather than through binding to specific sequence motifs. These ctenophore NRs may similarly regulate transcription of target genes in alternative ways by binding to non-canonical DNA sequences or secondary structures.

$D A X-1$ and SHP from vertebrates both function in molecular pathways as repressors. These NRs form dimers with other NRs, modulate the recruitment of cofactors, and repress transcriptional activity [41-43]. Whether the ctenophore NRs interact with other proteins or function as repressors requires additional research.

\section{Early divergence of the nuclear receptor superfamily}

Previous reports on the evolution of the nuclear receptor superfamily have concluded that the diversification of NRs in bilaterians occurred through two major radiation events $[1,44]$. The first wave occurred prior to the bilaterian ancestor, due to the presence of all six NR families in both representative deuterostomes and protostomes ([1], Figure 4). A second stage of diversification occurred within the vertebrate lineage, with the expansion of particular subfamilies due to gene or genome duplication events (or example, the steroidogenic family NR3C). However, the early diversification of the NR superfamily prior to the bilaterian ancestor has not been clarified despite the critical importance of these events to the understanding of the emergence of the six NR families present at the divergence of protostomes and deuterostomes.

This new classification of nuclear receptors, which is based on the full complement of sequences from four early branching phyla as well as bilaterian sequences, allows us to begin reconstructing the evolutionary events that led to the diversification of this superfamily (Figure 4). The first two branches of the animal tree (sponge and ctenophore) both contain NRs that cluster with subfamily NR2A (HNF4) and are most similar to these sequences. Sponges also have a second NR positioned between HNF4 and the rest of the NR superfamily. Thus, these genes suggest that HNF4 represents our best expectation for which NR was present in the ancestral metazoan. Reciprocally, the limited diversity of NRs from sponges and ctenophores provides additional evidence that these two phyla represent the first two branches from the animal tree. The next branch in the metazoan tree is represented by the placozoan Trichoplax and suggests a partial radiation in NR family 2 with the addition of NR2B (RXR) and NR2F (COUP-TF) 


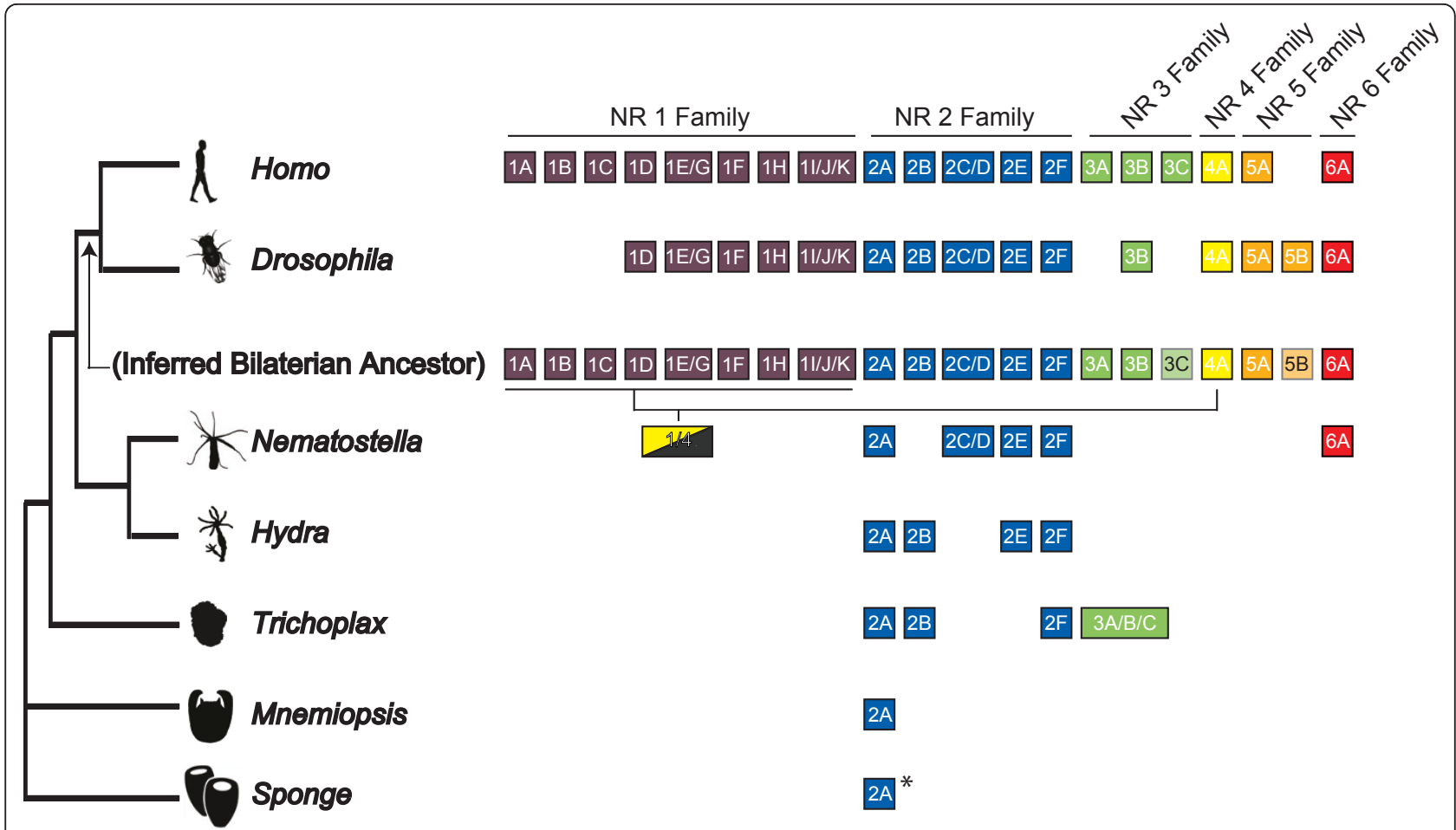

Figure 4 Evolutionary diversification of the NR superfamily in animals. At left is a cartoon of the metazoan tree showing the evolutionary relationships between ctenophores, sponges, placozoan, cnidarians, and bilaterians (that is, protostomes and deuterostomes). Due to current controversy about the branching order of the early diverging metazoans (see main text), the placement of lineages differs depending on particular analyses and thus an inference for the timing of origin and lineage-specific loss of particular NR families would vary. Colored boxes indicate a NR subfamily is represented by one or more genes for that species. The "inferred bilaterian ancestor" is based largely on a phylogenetic analysis conducted by Bertrand et al. [1]. However, two of these subfamilies are restricted to either protostomes (5B) or deuterostomes $(3 \mathrm{C})$ and we have shaded these and used black text to reflect a lack of conclusive support for the presence of these subfamilies in the bilaterian ancestor. Despite the absence of some genes in the D. melanogaster genome, studies of NRs from other protostomes (NR1A from Schistosoma mansoni [50] and NR3A from mollusks and annelids [51,52]) indicate that these subfamilies were present in the bilaterian ancestor and secondarily lost from Drosophila. Similarly, members of NR1B and 1C have been reported in mollusks and annelids [10], and thus are not restricted to deuterostomes. * The sponge Amphimedon queenslandica has two NRs, one that is supported as an ortholog to HNF4 (NR2A) and a second that groups between subfamily NR2A and the rest of the NR superfamily [see also [10]].

and the evolution of the first member of NR family 3 (as previously reported by [12]). The phylum Cnidaria is the closest sister group to the Bilateria from which NR sequences are currently available. From the two cnidarian species with complete genomes available (Nematostella vectensis and Hydra magnipapillata), we can infer that the cnidarian-bilaterian ancestor had a completely differentiated NR family 2, with NR2B (RXR) secondarily lost within the anthozoan lineage (see [5] for discussion). The class Anthozoa also has NRs that are best supported as NR family 6 (GCNF) and three class-specific paralogs that form an outgroup to NR families 1 and 4 in bilaterians. We hypothesize that the corresponding NRs were lost from Hydra, similar to a variety of other transcription factors (see [30]). Future characterization of NRs from other medusozoans, particularly members of the other two cnidarians classes Scyphozoa and Cubozoa, will allow additional testing of this hypothesis. Our data also support the hypothesis that the NR family 3 was lost early in the cnidarian lineage due to its presence in both Trichoplax and bilaterians. However, some phylogenetic analyses have suggested that Placozoa are the sister group to the Bilateria with the Cnidaria diverging from the stem earlier [for example, $[18,45]]$. If these are the correct phylogenetic relationships for these phyla, we would infer substantial NR loss from Trichoplax, including various members of NR family 2 .

This represents the first study to classify NRs from each early animal lineage in a single analysis. From our analyses, a number of NR families and subfamilies differentiated in the time between the divergence of the cnidarian lineage and the origin of the bilaterians. Most striking is the diversification of the NR 1 family into numerous subfamilies, at least eight of which were present in the bilaterian ancestor. Gaining insight into the diversification that occurred between these ancestors 
will depend on characterizing the gene complement of species that diverged during this time period. The best current candidates are the acoelomorphs, which are supported as a closer sister taxa to the bilaterians than the cnidarians $[13,46]$. Indeed, studies of the Hox complement from these species have provided insightful intermediates for characterizing the emergence of the homeobox family of transcription factors $[47,48]$.

By identifying NRs from these early branching metazoans with full genome sequences, we have additional power in characterizing the evolution of gene families, particularly since we are not inadvertently omitting genes missed in PCR-based surveys. For example, the original report of NRs from the coral Acropora identified ten NRs [3]. We queried newly published EST data from this species [23] and identified additional NRs that group in three families: the cnidarian-specific NR 1/4 family, NR2E, and NR2F. Because the Acropora sequences group with strong support with NRs from Nematostella, we expect that up to five more NRs are still not identified in this coral species.

With the additional cnidarian sequences and surveyed species, phylogenetic analyses suggest that NR evolution within the Cnidaria appears to have been a dynamic process with both gains (for example, duplication of $T R 2 / 4$, COUP-TF anthozoans) and losses (NR family 6 and NR family $1 / 4$ from either hydrozoans or ancestral lineage leading to the medusozoans, $R X R$ from anthozoans). Two subfamilies in NR family 2 (NR2E and F) have independently radiated at some point in this phylum's history due to the presence of multiple cnidarian paralogs. TR2/4 (NR2C/D) most likely duplicated within the anthozoans due to the presence of two homologs in the anemone Nematostella and the coral Acropora. The most dramatic cnidarian-specific radiation is represented by the duplications of the group of NRs sister to NR families 1 and 4 (see above). Together, these data support a hypothesis that NRs have undergone a handful of independent radiations within the Cnidaria. For one class of cnidarians, the Cubozoa, there is only one published NR sequence [9] and for another class, the Scyphozoa, there are no published NRs. Identifying additional NRs in these two classes will provide much needed data for assessing when particular NRs were duplicated and lost within the cnidarians. Finally, we have no evidence that similar radiations have occurred in ctenophores, sponges, or placozoans. This supports the conclusion that the NR radiations in the Cnidaria are unique among the early diverging animal lineages.

\section{Conclusion}

In this study, we have identified two NRs from the Mnemiopsis leidyi genome and one NR from Pleurobrachia pileus. All three ctenophore NRs contain the conserved
LBD but lack a conserved zinc-finger DBD, a domain that is conserved across all reported NRs in animals (with the exception of two genes unique to vertebrates). By applying a phylogenomic approach using NR sequences from organisms throughout the animal kingdom, we showed that ctenophores and sponges contain representatives of the same subfamily (NR2A), suggesting that the original NR was most similar to HNF4. The absence of the DBD from ctenophores may reflect an ancestral NR domain structure or a lineage-specific loss of this domain from an ancestral NR that contained both the DBD and LBD. Through analysis of NR family and subfamily representation in representative taxa, we conclude that the rate of diversification for the NR superfamily was fairly modest in the early diverging animals, similar to other gene families [49]. Additionally, several subfamilies underwent separate radiations in the phylum Cnidaria. Future work aimed at characterizing the function of the NRs from these early diverging phyla will enable tests of hypotheses regarding conserved and novel functions of members of this critical superfamily of transcription factors.

\section{Additional material}

Additional file 1: Primers used for 5- and 3-prime RACE of
Mnemiopsis leidyi NRs.
Additional file 2: Nuclear receptors from early diverging taxa used
for phylogenetic study of nuclear receptor superfamily. Table
showing gene names and accession numbers
Additional file 3: Protein sequences for NRs from Mnemiopsis leidyi
and Pleurobrachia pileus.
Additional file 4: Bayesian analysis of NR superfamily. Tree was
constructed using the identical alignment used for the maximum
likelihood analysis presented in Figure 3. Clades are annotated to family
and subfamily based on current nomenclature for the NR superfamily [8].
This tree is the consensus of four independent runs and was rooted with
the cluster containing the ctenophore sequences plus HNF4 from diverse
animals. Values above nodes indicate Bayesian support values. Posterior
probabilities below 0.7 were removed.

\section{Abbreviations}

BLAST: basic local alignment search tool; cDNA: complementary DNA; COUPTF: chicken ovalbumin upstream promoter transcription factor; DAX-1: dosage-sensitive sex reversal-adrenal hypoplasia congenital critical region on the X chromosome; DBD: DNA-binding domain; DNA: deoxyribonucleic acid; ERR: estrogen related receptor; EST: expressed sequence tag; FTZ: fushi tarazu; GCNF: germ cell nuclear factor; HMM: hidden Markov model; HNF4: hepatocyte nuclear factor 4; LBD: ligand-binding domain; NR: nuclear receptor; PCR: polymerase chain reaction; RACE: rapid amplification of CDNA ends; RNA: ribonucleic acid; RXR: retinoid " $X$ " receptor; SHP: small heterodimerization partner; UTR: untranslated region.

\section{Acknowledgements}

This research was supported by Award Number F32HD062178 from the Eunice Kennedy Shriver National Institute of Child Health \& Human Development (NICHD) to AMR, an NSF Graduate Research Fellowship to KP grants from the NSF and NASA to MQM, the Tropical Research Initiative of the Woods Hole Oceanographic Institution, and the Intramural Research 
Program of the National Human Genome Research Institute (NHGRI), National Institutes of Health. The content is solely the responsibility of the authors and does not necessarily represent the official views of the Eunice Kennedy Shriver National Institute of Child Health and Human Development or the National Institutes of Health.

\section{Author details}

${ }^{1}$ Biology Department, Woods Hole Oceanographic Institution, Woods Hole, MA, USA. ${ }^{2}$ Kewalo Marine Laboratory, Pacific Bioscience Research Center, University of Hawaii, Honolulu, HI, USA. ${ }^{3}$ Genome Technology Branch, National Human Genome Research Institute, National Institutes of Health, Bethesda, MD, USA.

\section{Authors' contributions}

AMR and AMT designed and conceived the study and drafted the manuscript. AMR also isolated Mnemiopsis nuclear receptors, performed gene annotation, and performed phylogenetic analyses. JFR identified Mnemiopsis nuclear receptors from the genomic assembly and contributed to phylogenetic analyses. KP isolated Mnemiopsis DNA and RNA and performed in situ hybridizations. JCM assembled the Mnemiopsis genome. ADB and MQM participated in the design of the study. All authors read and approved the final manuscript.

\section{Competing interests}

The authors declare that they have no competing interests.

Received: 29 July 2010 Accepted: 3 February 2011

Published: 3 February 2011

\section{References}

1. Bertrand S, Brunet F, Escriva H, Parmentier G, Laudet V, Robinson-Rechavi M: Evolutionary genomics of nuclear receptors: from twenty-five ancestral genes to derived endocrine systems. Molecular Biology and Evolution 2004, 21:1923-1937.

2. Gauchat D, Escriva H, Miljkovic-Licina M, Chera S, Langlois MC, Begue A, Laudet V, Galliot B: The orphan COUP-TF nuclear receptors are markers for neurogenesis from cnidarians to vertebrates. Developmental Biology 2004, 275:104-123.

3. Grasso L, Hayward D, Trueman J, Hardie K, Janssens P, Ball E: The evolution of nuclear receptors: evidence from the coral Acropora. Molecular Phylogenetics and Evolution 2001, 21:93-102.

4. Larroux C, Fahey B, Liubicich D, Hinman V, Gauthier M, Gongora M, Green K, Wörheide G, Leys S, Degnan B: Developmental expression of transcription factor genes in a demosponge: insights into the origin of metazoan multicellularity. Evolution and Development 2006, 8:150-173.

5. Reitzel AM, Tarrant AM: Nuclear receptor complement of the cnidarian Nematostella vectensis: phylogenetic relationships and developmental expression patterns. BMC Evolutionary Biology 2009, 9:230.

6. Wiens M, Batel R, Korzhev M, Müller W: Retinoid X receptor and retinoic acid response in the marine sponge Suberites domuncula. Journal of Experimental Biology 2003, 206:3261-3271.

7. Phelps C, Gburcik V, Suslova E, Dudek P, Forafonov F, Bot N, MacLean M, Fagan RJ, Picard D: Fungi and animals may share a common ancestor to nuclear receptors. 2006, 103:7077-7081.

8. Nuclear Receptor Nomenclature Committee: A Unified Nomenclature System for the Nuclear Receptor Superfamily. Cell 1999, 97:161-163.

9. Kostrouch Z, Kostrouchova M, Love W, Jannini E, Piatigorsky J, Rall J: Retinoic acid X receptor in the diploblast, Tripedalia cystophora. Proceedings of the National Academy of Science, USA 1998, 95:13442-13447.

10. Bridgham JT, Eick GN, Larroux C, Deshpande K, Harms MJ, Gauthier ME, Ortlund EA, Degnan BM, Thornton JW: Protein evolution by molecular tinkering: diversification of the nuclear receptor superfamily from a ligand-dependent ancestor. PLOS Biology 2010, 8:e1000497.

11. Srivastava M, Begovic E, Chapman J, Putnam NH, Hellsten U, Kawashima T, Kuo A, Mitros T, Salamov A, Carpenter ML, Signorovitch AY, Moreno MA Kamm K, Grimwood J, Schmutz J, Shapiro H, Grigoriev IV, Buss LW, Schierwater B, Dellaporta SL, Rokhsar DS: The Trichoplax genome and the nature of placozoans. Nature 2008, 454:955-960.

12. Baker ME: Trichoplax, the simplest known animal, contains an estrogenrelated receptor but no estrogen receptor: Implications for estrogen receptor evolution. Biochemical and Biophysical Research Communications 2008, 375:623-627.
13. Hejnol A, Obst M, Stamatakis A, Ott M, Rouse GW, Edgecombe GD, Martinez P, Baguna J, Bailly X, Jondelius U, Wiens M, Müller WE, Seaver E, Wheeler WC, Martindale MQ, Giribet G, Dunn CW: Assessing the root of bilaterian animals with scalable phylogenomic methods. Proceedings of the Royal Society B: Biological Sciences 2009, 276:4261-4270.

14. Dunn CW, Hejnol A, Matus DQ, Pang K, Browne WE, Smith SA, Seaver E, Rouse GW, Obst M, Edgecombe GD, Sørensen MV, Haddock SH, SchmidtRhaesa A, Okusu A, Kristensen RM, Wheeler WC, Martindale MQ, Giribet G: Broad phylogenomic sampling improves resolution of the animal tree of life. Nature 2008, 452:745-749.

15. Schierwater B, Eitel M, Jakob W, Osigus H Jr, Hadrys H, Dellaporta SL, Kolokotronis SO, DeSalle R: Concatenated analysis sheds light on early metazoan evolution and fuels a modern "urmetazoan" hypothesis. PLOS Biology 2009, 7:e1000020.

16. Philippe H, Derelle R, Lopez P, Pick K, Borchiellini C, Boury-Esnault N, Vacelet J, Renard E, Houliston E, Quéinnec E, Da Silva C, Wincker P, Le Guyader H, Leys S, Jackson DJ, Schreiber F, Erpenbeck D, Morgenstern B, Wörheide G, Manuel M: Phylogenomics revives traditional views on deep animal relationships. Current Biology 2009, 19:706-712.

17. Peterson KJ, Eernisse DJ: Animal phylogeny and the ancestry of bilaterians: inferences from morphology and 18S rDNA gene sequences. Evolution \& Development 2001, 3:170-205.

18. Pick KS, Philippe $H$, Schreiber F, Erpenbeck D, Jackson DJ, Wrede P, Wiens M, Alie A, Morgenstern B, Manuel M, Wörheide G: Improved phylogenomic taxon sampling noticeably affects non-bilaterian relationships. Mol Biol Evol 2010, 27:1983-1987.

19. Wallberg A, Thollesson M, Farris JS, Jondelius U: The phylogenetic position of the comb jellies (Ctenophora) and the importance of taxonomic sampling. Cladistics 2004, 20:558-578.

20. Podar M, Haddock SHD, Sogin ML, Harbison GR: A molecular phylogenetic framework for the phylum Ctenophora using 18S rRNA genes. Molecular Phylogenetics and Evolution 2001, 21:218-230.

21. Ryan JF, Pang K, Program NCS, Mullikin JC, Martindale MQ, Baxevanis AD: The homeodomain complement of the ctenophore Mnemiopsis leidyi suggests that Ctenophora and Porifera diverged prior to the ParaHoxozoa. EvoDevo 2010, 1:9.

22. Pang K, Martindale MQ: Developmental expression of homeobox genes in the ctenophore Mnemiopsis leidyi. Development Genes and Evolution 2008, 218:307-319.

23. Meyer E, Aglyamova G, Wang S, Buchanan-Carter J, Abrego D, Colbourne J, Willis B, Matz M: Sequencing and de novo analysis of a coral larval transcriptome using 454 GSFlx. BMC Genomics 2009, 10:219.

24. Edgar R: MUSCLE: multiple sequence alignment with high accuracy and high throughput. Nucleic Acids Research 2004, 32:1792-1797.

25. Stamatakis A: RAxML-VI-HPC: Maximum likelihood-based phylogenetic analyses with thousands of taxa and mixed models. Bioinformatics 2006, 22:2688-2690.

26. Huelsenbeck JP, Ronquist F: MRBAYES: Bayesian inference of phylogenetic trees. Bioinformatics 2001, 17:754-755.

27. Thornton JW: Nuclear receptor diversity: phylogeny, evolution and endocrine disruption. Pure and Applied Chemistry 2003, 75:1827-1839.

28. Laudet $V$ : Early diversification of the nuclear receptor superfamily: early diversification from an ancestral orphan receptor. Journal of Molecular Endocrinology 1997, 19:207-226.

29. Tarrant AM, Cortes J, Atkinson M, Atkinson S, Johanning K, Chiang TC, Vargas JA, McLachlan JA: Three orphan nuclear receptors in the scleractinian coral Pocillopora damicornis from the Pacific coast of Costa Rica. Rev Biol Trop 2008, 56:39-48.

30. Chapman JA, Kirkness EF, Simakov O, Hampson SE, Mitros T, Weinmaier T, Rattei T, Balasubramanian PG, Borman J, Busam D, et al: The dynamic genome of Hydra. Nature 2010, 464:592-596.

31. Escriva H, Safi R, Hanni C, Langlois MC, Saumitou-Laprade P, Stehelin D, Capron A, Pierce R, Laudet V: Ligand binding was acquired during the evolution of nuclear receptors. Proceedings of the National Academy of Science, USA 1997, 94:6803-6808.

32. Brosius J: The contribution of RNAs and retroposition to evolutionary novelties. Genetica 2003, 118:99-115.

33. Wu W, Niles EG, Hirai H, Loverde PT: Evolution of a novel subfamily of nuclear receptors with members that each contain two DNA binding domains. BMC Evolutionary Biology 2007, 7:27.

34. Giguere V: Orphan nuclear receptors: from gene to function. Endocrine Reviews 1999, 20:689-725. 
35. Park YY, Teyssier C, Vanacker JM, Choi HS: Distinct repressive properties of the mammalian and fish orphan nuclear receptors SHP and DAX-1. Molecules and Cells 2007, 23:331-339.

36. Nauber U, Pankratz MJ, Kienlin A, Seifert E, Klemm U, Jackle H: Abdominal segmentation of the Drosophila embryo requires a hormone receptorlike protein encoded by the gap gene knirps. Nature 1988, 336:489-492.

37. Sluder A, Matthews S, Hough D, Yin V, Maina C: The nuclear receptor superfamily has undergone extensive proliferation and diversification in nematodes. Genome Research 1999, 9:103-120.

38. Näär AM, Thakur JK: Nuclear receptor-like transcription factors in fungi. Genes \& Development 2009, 23:419-432.

39. Khorasanizadeh S, Rastinjad F: Nuclear-receptor interactions on DNAresponse elements. Trends Biochem Sci 2001, 26:384-390.

40. Zazopoulos E, Lalli E, Stocco DM, Sassone-Corsi P: DNA binding and transcriptional repression by DAX-1 blocks steroidogenesis. Nature 1997, 390:311-315

41. Crawford PA, Dorn C, Sadovsky Y, Milbrandt J: Nuclear receptor DAX-1 recruits nuclear receptor corepressor N-CoR to steroidogenic factor 1. Molecular and Cellular Biology 1998, 18:2949-2956.

42. Song KH, Park YY, Park KC, Hong CY, Park JH, Shong M, Lee K, Choi HS: The atypical orphan nuclear receptor DAX-1 interacts with orphan nuclear receptor Nur77 and represses its transactivation. Molecular Endocrinology 2004, 18:1929-1940.

43. Masuda N, Yasumo H, Tamura T, Hashiguchi N, Furusawa T, Tsukamoto T, Sadano H, Osumi T: An orphan nuclear receptor lacking a zinc-finger DNA-binding domain: interaction with several nuclear receptors. Biochimica et Biophysica Acta (BBA) - Gene Structure and Expression 1997, 1350:27-32.

44. Laudet V, Hanni V, Coll J, Catzeflis F, Stehelin D: Evolution of the nuclear receptor gene superfamily. The EMBO Journal 1992, 11:1003-1013.

45. Collins AG: Evaluating multiple alternative hypotheses for the origin of Bilateria: An analysis of $18 \mathrm{~S}$ rRNA molecular evidence. Proceedings of the National Academy of Sciences of the United States of America 1998, 95:15458-15463.

46. Ruiz-Trillo I, Riutort M, Littlewood DTJ, Herniou EA, Baguñà J: Acoel flatworms: earliest extant bilaterian metazoans, not members of platyhelminthes. Science 1999, 283:1919-1923.

47. Hejnol A, Martindale M: Coordinated spatial and temporal expression of Hox genes during embryogenesis in the acoel Convolutriloba longifissura. BMC Biology 2009, 7:65.

48. Cook CE, Jiménez E, Akam M, Saló E: The Hox gene complement of acoel flatworms, a basal bilaterian clade. Evolution \& Development 2004, 6:154-163.

49. Larroux C, Luke GN, Koopman P, Rokhsar DS, Shimeld SM, Degnan BM: Genesis and expansion of metazoan transcription factor gene classes. Molecular Biology and Evolution 2008, 25:980-996.

50. Wu W, Niles E, LoVerde P: Thyroid hormone receptor orthologues from invertebrate species with emphasis on Schistosoma mansoni. BMC Evolutionary Biology 2007, 7:150

51. Keay J, Bridgham JT, Thornton JW: The Octopus vulgaris estrogen receptor is a constitutive transcriptional activator: evolutionary and functional implications. Endocrinology 2006, 147:3861-3869.

52. Keay J, Thornton JW: Hormone-activated estrogen receptors in annelid invertebrates: Implications for evolution and endocrine disruption. Endocrinology 2009, 150:1731-1738.

doi:10.1186/2041-9139-2-3

Cite this article as: Reitzel et al:: Nuclear receptors from the ctenophore Mnemiopsis leidyi lack a zinc-finger DNA-binding domain: lineagespecific loss or ancestral condition in the emergence of the nuclear receptor superfamily? EvoDevo 2011 2:3.

\section{Submit your next manuscript to BioMed Central and take full advantage of:}

- Convenient online submission

- Thorough peer review

- No space constraints or color figure charges

- Immediate publication on acceptance

- Inclusion in PubMed, CAS, Scopus and Google Scholar

- Research which is freely available for redistribution

Submit your manuscript at www.biomedcentral.com/submit 